\title{
A QUALIDADE CROMÁTICA PERCEBIDA EM ESCRITÓRIOS
}

\author{
MELLO FERNANDES, Manuela (1) \\ COSTA FILHO, Lourival (2) \\ (1) UFPE | PPGDESIGN | CAC | Mestranda em Design \\ e-mail: manuelafernandes02@gmail.com \\ (2) UFPE | PPGDESIGN | CAC | Doutor em Desenvolvimento Urbano \\ e-mail: lourivalcosta@yahoo.com
}

\begin{abstract}
RESUMO
Este artigo irá apresentar proposta para a avaliação da qualidade cromática percebida em escritórios a partir da visão de especialistas e não-especialistas no projeto desses espaços de trabalho. A investigação empírica, estruturada através da Teoria das Facetas, propõe o Sistema de Classificações Múltiplas para coletar os dados e o procedimento não métrico e multidimensional conhecido como Análise da Estrutura de Similaridade (Similarity Structure Analysis) para interpretá-los. Espera-se que as evidências empíricas possam confirmar as hipóteses inicialmente formuladas, revelar conceitos envolvidos e sua estrutura interna, a forma como eles se inter-relacionam no tipo de avaliação proposto, além da falta de consenso dos resultados entre os dois diferentes grupos participantes.
\end{abstract}

Palavras-chaves: escritório; qualidade cromática; ergonomia do ambiente construído.

\begin{abstract}
This article aims to present a proposal for the assessment of the perceived chromatic quality in offices from the perspective of experts and non-experts in the design of these workspaces. Facet Theory was chosen for the structure of the empirical research. A Multiple Sortings Procedure was selected as the tool to collect the data, and the non-metric multidimensional procedure of Smillarity Structure Analysis was used to interpret it. It is hoped that the empirical evidence will confirm the hypotheses proposed, and unearth the concepts involved, their internal structures, and the way they interrelate in this type of evaluation, as well as the lack of consensus between the groups which were sampled.
\end{abstract}

Keywords: office; chromatic quality; ergonomic of the built environment.

\section{INTRODUÇÃO}

Este artigo apresenta uma pesquisa que está em andamento para o desenvolvimento de dissertação de mestrado em design, na linha de pesquisa da ergonomia, mais precisamente na área da ergonomia do ambiente construído.

A pesquisa envolve o efeito da cor em ambientes de trabalho em escritórios que, de acordo com a visão de Reis e Moraes (2004) para os espaços de trabalho, podem ser descritos como conjuntos de sistemas de áreas que se sucedem, se organizam dentro do edifício e que provavelmente participam como agentes de formação das condições de trabalho de seus ocupantes, gerando o ambiente de trabalho organizacional. 


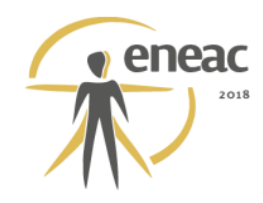

Ainda segundo as autoras, esse ambiente se relaciona aos fenômenos existenciais que se identificam com elementos espaciais. Esses fenômenos interferem, interagem e atuam no desempenho do trabalhador, na realização de suas tarefas no ambiente de trabalho. Partindo da ordenação desses elementos no espaço para o desenvolvimento de atividades, pressupõe-se atuação e cuidado com esse espaço.

A cor, nos locais de trabalho de escritórios, apresenta-se como um dos elementos ambientais que, atuando como estímulo, pode provocar sensações/percepções e promover emoções (FIGUEIREDO; MONT'ALVÃO, 2004). Sendo assim, segundo Mahnke (1996), tem grande impacto nas reações psicológicas e no bem-estar fisiológico, podendo afetar o organismo humano em bases tanto visuais quanto não visuais. Em suma, para o autor, a resposta humana para a cor é total, já que ela influencia tanto psicológica como fisiologicamente.

A partir dessas influências, é possível afirmar que a cor pode ser utilizada para auxiliar os trabalhadores de escritórios a se sentirem física e emocionalmente mais confortáveis. Nesse contexto, atua, através de seus efeitos psicofísicos, positiva ou negativamente nos resultados e comportamentos desses trabalhadores. Logo, as interações humanas com 0 ambiente de trabalho e os elementos espaciais, a partir de suas sensações e percepções, influenciam o comportamento, interferindo diretamente na tão buscada produtividade e na saúde dos trabalhadores.

A escolha da cor, ao induzir sentimentos de conforto, deve estar adequada às necessidades psicofísicas dos trabalhadores para o desenvolvimento de suas atividades nos ambientes considerados (MAHNKE, 1996). Em ambientes de trabalho em escritórios, porém, tal decisão é muitas vezes baseada no gosto dos que decidem e/ou realizam o projeto cromático, na facilidade de manutenção ou até mesmo nas cores da identidade coorporativa da empresa.

Apesar de profissionais da área de projeto teorizarem sobre a qualidade visual, as análises empíricas sobre a qualidade cromática percebida em ambientes de trabalho de escritório têm ficado para trás. Somando-se a isso, há lacuna na literatura que versa sobre esse objeto de estudo, cuja maior produção publicada refere-se ao planejamento e a execução de arranjos físicos do espaço de trabalho, que resumem o aspecto organizacional do trabalho.

Exposto o problema da pesquisa em andamento, admite-se ser relevante responder: quais características dos ambientes de trabalho de escritório são aderentes para a avaliação de sua qualidade cromática percebida? Quais efeitos emocionais que essas características desencadeiam nos trabalhadores? Há consenso dos resultados entre os grupos abordados?

Buscando responder a essas questões da pesquisa, delineou-se como objetivo geral avaliar a qualidade cromática percebida nos ambientes de trabalho de escritório a partir da percepção de especialistas e não especialista no projeto desses espaços. Considerando-se os dois diferentes grupos escolhidos como recorte participativo, bem como a cidade do Recife como recorte espacial para a investigação empírica, elencam-se ainda como objetivos específicos: 1| testar a aderência de certas características visuais dos ambientes de trabalho de escritório para a avaliação da qualidade visual percebida; 2| verificar a influência dessas características nos aspectos de prazer, excitação e relaxamento nesses ambientes; 3| analisar o consenso dos resultados entre os dois diferentes grupos abordados.

Quando se toma a interação entre trabalhador e ambientes de trabalho de escritório, mais especificamente sobre o efeito da cor na qualidade visual percebida nesses ambientes, é importante adotar uma abordagem interdisciplinar para responder a esse objetivo. Assim, imaginou-se que a associação da estética ambiental (psicologia ambiental/estética empírica) com a ergonomia do ambiente construído pode favorecer esse enfoque, a partir do interesse dessas áreas em relação ao modo como as pessoas percebem e tomam decisões no ambiente. $O$ estudo dessa relação pode levar a uma melhor compreensão sobre a cor em ambientes de trabalho de escritórios, fornecendo bases objetivas sobre a qualidade visual percebida nesses espaços e, mais amplamente, sua melhoria. 


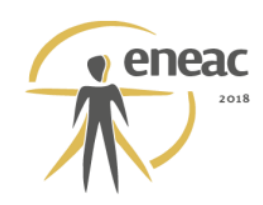

\section{CONSIDERAÇÕES TEÓRICAS}

A maneira como o tema será apresentado nesta seção, coloca a abordagem do objeto de estudo teórico antes do empírico, ou seja, primeiro trazem-se conceitos relacionados com o tipo de avaliação subjetiva que se pretende realizar e uma breve exposição sobre escritórios, enfatizando suas relações com a ergonomia do ambiente construído. Em seguida, faz-se uma rápida introdução aos aspectos da cor como elemento ambiental. Os aportes teóricos e conceituais, quando aprofundados na pesquisa proposta, visam fundamentar as discussões dos resultados empíricos obtidos. Neste item, tem o principal propósito de justificar escolhas metodológicas, à medida que a pesquisa vai sendo desenhada nos próximos itens.

Esta seção será iniciada com os aspectos teóricos relacionados às avaliações psicológicas, portanto subjetivas, sobre a qualidade visual percebida em ambientes de trabalho de escritório. Tais avaliações têm referência primária para os elementos ambientais (julgamentos perceptuais/cognitivos) ou para os sentimentos das pessoas sobre o ambiente (julgamentos emocionais). Como resultado, a qualidade visual percebida será medida nesta pesquisa através de julgamentos avaliativos de ambientes de trabalho de escritórios.

Em relação as avaliações subjetivas, é importante ressaltar que, segundo Nasar (2000), os elementos ambientais têm impacto importante na experiência humana; podem evocar fortes emoções como agrado ou desagrado, atuar como efeito estressor ou restaurador e fazer inferências sobre lugares e pessoas. Podem também influenciar o comportamento humano, como a decisão de frequentar ou de evitar certos lugares. E, como as pessoas respondem as inferências derivadas de estímulos visuais e não-visuais de lugares, a qualidade visual do entorno de uma pessoa tem efeitos poderosos sobre sua experiência.

Embora a qualidade visual percebida dependa, em parte, de fatores perceptuais/cognitivos (como a avaliação da variedade de cores de uma cena), é, por definição, um julgamento emocional (como a avaliação da agradabilidade de uma cena), que envolve avaliação e sentimentos (NASAR, 1998).

Para serem relevantes, tais julgamentos, devem centrar-se nas avaliações afetivas para os ambientes que, para Russel (1988), ocorrem quando uma pessoa julga algo como tendo uma qualidade afetiva, como sereno, intenso, e assim por diante. Examinando a questão, o autor definiu quatro dimensões para sintetizar todos os termos descritores da avaliação afetiva: agradável, estimulante, emocionante, relaxante. Ainda conforme destaca, o agrado é uma dimensão puramente avaliativa e o estímulo independe da dimensão avaliativa. A emoção e o relaxamento envolvem misturas de avaliação e estímulo. Logo, as pessoas experienciam um ambiente emocionante como mais agradável e estimulante do que os entediantes; e os ambientes relaxantes como mais agradável e menos estimulante do que os aflitivos.

Apesar das respostas avaliativas, por si só, não poderem prever o comportamento real, a avaliação combinada de respostas avaliativas e o comportamento previsto dá uma boa indicação do comportamento real (NASAR, 1988). Dessa forma, na pesquisa proposta, os participantes serão solicitados a avaliar em que medida várias cenas de ambientes de trabalho de escritório favorecem a agradabilidade, a emoção e o relaxamento.

Duas características dos elementos de ambientes de escritório, coerência e complexidade, foram escolhidas para estudo, por causa de suas prováveis influências para a qualidade visual percebida. A coerência é definida como o grau em que a cena se encaixa e a complexidade é definida como a quantidade de variedade na cena.

De acordo com Kaplan (1988), as pessoas preferem ambientes que oferecem envolvimento e fazem sentido. Assim, a coerência contribui para a preferência por tornar o ambiente compreensível, já a complexidade por envolver o observador. 


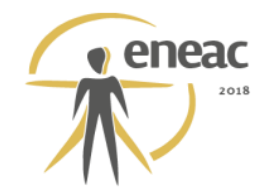

Auxiliando a compreensão, a coerência - obtida pela redução do contraste dos elementos na cena - pode reduzir a incerteza e aumentar o tom hedônico (prazer ou beleza) (WOHLWILL, 1976; KAPLAN; KAPLAN, 1982).

Por definição, a complexidade cria incerteza, que, por sua vez, provoca o envolvimento. Pouca complexidade é monótona e cansativa; muita é caótica e estressante. O nível médio de complexidade parece ser o mais agradável, ou o ideal. Logo, o tom hedônico da cena tem sido postulado como tendo a forma de "U" invertido em relação à complexidade (BERLYNE, 1972; WOHLWILL, 1976).

Igualmente importante para esta pesquisa é o da ergonomia e como essa disciplina se relaciona com a cor nos ambientes de trabalho de escritório. A ergonomia do ambiente construído, na visão de Silva Júnior e Costa Filho (2016), se interessa em estudar as interações dos usuários com o ambiente construído, contemplando tanto a consideração de aspectos de ordem física quanto a identificação da percepção dos usuários a partir de uma abordagem sistêmica (humano-atividade-ambiente) ampla e sob o prisma desse usuário.

Ao abordar as condições necessárias para o projeto de ambientes, Bins Ely (2004) afirma a importância de conhecer os elementos do ambiente que podem causar estímulos sensoriais (perceber e receber informações) e provocar respostas ao nível do corpo, traduzidas no comportamento. Ainda segundo a autora, som, cor, aroma, textura e forma são alguns exemplos de elementos que podem provocar sensações e promover bem-estar emocional.

As interações do indivíduo com o ambiente a partir de suas sensações e percepções, interferem na sua forma de agir (MONT'ALVÃO; FIGUEIREDO, 2004). No caso, interferem na decisão de frequentar e permanecer nos ambientes de trabalho de escritórios, além de exercerem efeitos sobre outras variáveis comportamentais derivadas de suas qualidades percebidas como agrado/desagrado, estimulo/enfado, emoção/chatice, relaxamento/aflição.

Há de se ressaltar que, apoiando-se em Figueiredo e Mont'Alvão (2006), cada vez mais as pessoas passam o dia em seus ambientes de trabalho. A fim de minimizar erros e evitar monotonia por parte dos funcionários, esses ambientes precisam ser estimulantes. Desta forma, é importante estudar como trazer atratividade para esses locais a fim de promover mais estímulos e satisfação aos funcionários.

O compromisso com o espaço de trabalho, como fica demonstrado, é muito grande, pois, segundo Reis e Moraes (2004), esse não é um espaço de pequena permanência nem de poucas trocas, mas um espaço onde seus ocupantes estão em torno de oito horas, praticamente todos os dias, durante a maior parte do tempo de suas vidas e em atividade constante, física e cognitiva.

Quanto a preocupação com a cor nos ambientes de trabalho, Silva e Bormio (2016) reforça que entre os fatores físicos que interferem no ambiente, a cor destaca-se pela capacidade de influenciar os seres humanos.

De acordo com o tipo da tarefa realizada, a cor do ambiente pode ter efeitos também sobre o desempenho e a percepção das tarefas. Dentro dessa perspectiva, determinar os efeitos da cor nos ambientes de trabalho em escritórios sobre os trabalhadores pode ser útil para o projeto ergonômico desses ambientes e, no próximo item, inicia-se a apresentação das considerações teórico-metodológicas que auxiliou a estruturação da investigação empírica.

\section{CONSIDERAÇÕES TEÓRICO-METODOLÓGICAS}

Esta pesquisa adotou a Teoria das Facetas (TF) no desenho de sua investigação empírica (GUTTMAN, 1981; SHYE, ELIZUR \& HOFFMAN, 1994; BILSKY, 2003), apoiando-se também na "avaliação objetivada do lugar", abordada por Canter (1983), que considera a 


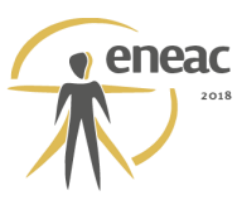

avaliação ambiental relacionada à extensão dos propósitos e das ações dirigidas aos objetivos humanos que recaem nos ambientes e, como tal, leva em conta as intenções das pessoas nos ambientes.

O uso da TF envolve inicialmente a identificação dos diferentes conceitos ou dimensões que delineiam a pesquisa, e podem advir da literatura ou de explorações in loco. Essa etapa consiste em estabelecer hipóteses, encontrar as facetas do modelo teórico e definir os elementos que as constituem. Cada faceta representa uma categoria conceitual, constituída por subcategorias de elementos a serem pesquisados (COSTA FILHO, 2014).

Por definição, normalmente, existem três facetas básicas de avaliação ambiental, cada uma representando um componente do lugar investigado: referente, foco, nível. A primeira faceta define o referente da experiência e expõe os diferentes aspectos em que as pessoas se baseiam para realizar suas avaliações. A faceta do foco modula o referente da experiência. A faceta do nível leva em conta a existência da escala ambiental, que influi na avaliação dos espaços. Essas relações entre os diversos aspectos da experiência das pessoas com um determinado ambiente podem ser sumarizadas através de uma sentença estruturadora, que descreve os componentes dos ambientes e a forma como são vivenciados pelos usuários.

Cabe agora apresentar a aplicação da Teoria das Facetas nesta pesquisa. O Quadro 1 propõe a sentença estruturadora para a avaliação da qualidade cromática percebida em ambientes de trabalho de escritório, os nomes das facetas de conteúdo e seus elementos de composição interna. Essa sentença é uma expressão da qualidade visual percebida de para esses tipos de ambiente. $O$ primeiro tipo de faceta se refere à população abordada. $O$ segundo tipo abrange o conteúdo das variáveis pesquisadas e, juntamente com a faceta da população abordada, define o domínio desta pesquisa. $O$ terceiro tipo descreve o universo de respostas possíveis (racional) em relação ao domínio da pesquisa.

\section{Quadro 01: Sentença estruturadora para avaliação da qualidade cromática percebida em ambientes de trabalho de escritórios.}

\begin{tabular}{|c|c|c|}
\hline \multicolumn{3}{|c|}{ A pessoa x (especialista | não especialista) avalia que ambientes de trabalho de escritórios com } \\
\hline $\begin{array}{l}\text { faceta (a) - referente } \\
\text { CONTRASTE [COR] }\end{array}$ & & $\begin{array}{l}\text { faceta (b) - referente } \\
\text { COMPLEXIDADE [COR] }\end{array}$ \\
\hline $\begin{array}{l}\text { (a1) baixo } \\
\text { (b1) médio } \\
\text { (c1) alto }\end{array}$ & com & $\begin{array}{l}\text { (b1) mínima } \\
\text { (b2) moderada } \\
\text { (b3) máxima }\end{array}$ \\
\hline $\begin{array}{l}\text { RACIONAL } \\
\text { (1) nada } \\
\text { (2) pouco } \\
\text { (3) mais ou menos } \\
\text { (4) muito } \\
\text { (5) muitíssimo }\end{array}$ & a agr & a emoção e o relaxamento percebidos nesses espaços \\
\hline
\end{tabular}

Fonte: autores da pesquisa

A população que se propõe avaliar nesta pesquisa é constituída por dois diferentes tipos de interesse nos ambientes de trabalho de escritório. No primeiro grupo, predomina o interesse de projetar os espaços e/ou os arranjos físicos do espaço de trabalho, sendo sua experiência com esse tipo de espaço marcado pelo conhecimento científico. No segundo, há o interesse de trabalhar e sua experiência com os ambientes é marcado pelo senso comum. Fazem parte desses grupos: 1| especialistas; 2| não especialistas 
As duas facetas de conteúdo, relacionadas com a qualidade cromática percebida de ambientes de trabalho de escritório - contraste e complexidade - foram consideradas hipoteticamente importantes para a avaliação pretendida. Todas são facetas de referente da experiência e representam características ambientais que podem ser tomadas para a avaliação da qualidade cromática percebida. A faceta A - CONTRASTE - está associada aos diferentes níveis - baixo, médio, alto - em que as cores contrastam nos ambientes de trabalho de escritório. A faceta $B$ - COMPLEXIDADE - está relacionada com o número de elementos - mínimo, moderado, máximo - notavelmente coloridos nas cores. O foco e o nível da experiência não foram considerados como facetas de conteúdo, na medida em que o foco foi relacionado com os elementos internos das duas facetas de referente e o nível das avaliações é geral, ou seja, em ambientes de trabalho de escritório.

O racional, que descreve as possíveis respostas da população à qualidade cromática percebida de ambientes de trabalho de escritório, tem 5 intervalos: 1 | nada; 2| pouco; 3| mais ou menos; 4| muito; $5 \mid$ demais.

A sentença estruturadora de avaliação da qualidade cromática percebida de ambientes de trabalho de escritório aqui proposta, como uma referência inicial da pesquisa, é analisada em relação aos resultados empíricos que devem confirmar ou refutar as hipóteses levantadas. Logo, após a análise/interpretação dos dados, há informações suficientes para construir ou não uma nova sentença, a ser estruturada como uma consequência direta dos resultados empíricos apurados.

\section{CONSIDERAÇÕES METODOLÓGICAS}

Nesta seção expõem-se as considerações metodológicas esboçadas para a investigação empírica. O desenvolvimento da metodologia, todavia, é parte integrante da pesquisa que está em andamento e precisa ser submetida a testes de consistência antes de ser tomada como definitiva.

Para a coleta dos dados, optou-se pelo Sistema de Classificações Múltiplas (CANTER; BROWN; GROAT, 1985), que consiste em solicitar informações aos participantes para classificar os mesmos elementos diversas vezes, com a finalidade de compreender suas ideias sobre o objeto de estudo.

O Sistema de Classificações Múltiplas vem sendo muito utilizado para explorar experiências ambientais (COSTA FILHO, 2014), e sua aplicabilidade foi ampliada por permitir o uso de ilustrações e outros materiais visuais difíceis de acomodar dentro de outros instrumentos.

A pesquisa adotará como elementos de estímulo, para serem classificados, um conjunto de fotografias de ambientes de trabalho de escritório. A geração desse conjunto, contudo, precisa estar diretamente associada às variáveis desta pesquisa, todas listadas na sentença estruturadora para a avaliação da qualidade cromática percebida de ambientes de trabalho de escritório, que estabelece precisamente a relação entre todas as partes envolvidas, ou seja, a população amostral, o que se pretende avaliar, os referentes, o foco e o nível da experiência, bem como o racional comum às respostas.

Pretende-se utilizar, junto aos participantes, três "classificações dirigidas". Nesse tipo de classificação, solicita-se que o entrevistado classifique os elementos conforme critérios preestabelecidos. Na primeira classificação, que busca responder ao primeiro objetivo específico da pesquisa, os respondentes serão convidados a indicarem em que medidas as cenas de ambientes de trabalho de escritório favorecem: (i) a agradabilidade; (ii) a excitação; (iii) o relaxamento, e têm a finalidade de responder aos objetivos geral e específicos da pesquisa em desenvolvimento. 


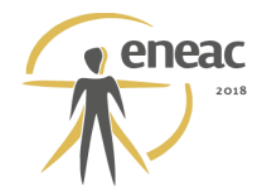

As classificações realizadas serão registradas em um formulário especialmente elaborado. Como todos os participantes abordados serão submetidos aos mesmos procedimentos, os dados que variam limitam-se às informações específicas de cada grupo (especialistas - não especialistas), restritas à primeira página do modelo proposto.

Conforme apurado por Stamps (1992), quando se trata da avaliação da qualidade visual do ambiente, pode-se obter resultados muito confiáveis ao utilizar, como elementos de estímulo, fotografias coloridas, vídeos, slides, fotomontagens e simulações.

Os dados obtidos nas classificações dirigidas serão interpretados através do procedimento não-métrico e multidimensional da SSA (Smilarity Structure Analysis), executado com o auxílio do programa informático HUDAP (Hebrew University Data Analysis Package).

A SSA, de acordo com Roazzi, Monteiro e Rullo (2009), é um sistema de escalonamento multidimensional concebido para analisar a matriz de correlações entre " $n$ " variáveis representadas graficamente como pontos num espaço euclidiano. O sistema fundamenta-se no princípio da contiguidade que, como tal, traduz as relações de similaridades entre itens, configurado pelas distâncias entre os pontos. Isso significa que a proximidade das variáveis no espaço multidimensional é proporcional ao grau de correlação que apresentam. Essas relações de similaridades podem formar regiões de contiguidade que possibilitam verificar se as hipóteses iniciais são transformadas em hipóteses regionais, em relação às quais se espera evidenciar regiões que abarquem aos elementos internos de cada faceta.

A solução da SSA propriamente dita compreende um mapeamento de todos os itens processados para um espaço de dimensionalidade especificada. Na pesquisa, essa solução compreenderá o processamento das cenas de ambientes de trabalho de escritório avaliadas por cada participante para um espaço bidimensional (diagrama do espaço da SSA).

As análises dos diagramas da SSA podem revelar relações e regras implícitas nos dados obtidos, imperceptíveis nas análises quantitativas usuais. Ao final dos testes, os resultados fornecem bases para a confirmação da sentença estruturadora ou para a construção de uma nova, com a redefinição das hipóteses inicialmente consideradas.

Caso as hipóteses regionais sejam verificadas, revelam aspectos relativamente estáveis do conceito investigado, dando-lhe legitimidade, além de confirmar a estrutura interna de conceitos e atributos, possibilitando a percepção de componentes empiricamente verificáveis e da forma como se inter-relacionam (SHYE; ELIZUR; HOFFMAN, 1994).

A SSA permite ainda testar se um determinado grupo opera da mesma maneira que outro na avaliação de vitrinas, questão relacionada com o terceiro objetivo formulado na pesquisa. Tal método, segundo Monteiro e Roazzi (2009), é considerado um grande avanço na SSA e permite a integração de subpopulações no mapa de componentes originais. Logo, em vez de analisar diversas projeções diferentes, produzidas para cada grupo considerado em uma pesquisa, é possível apreciar uma única projeção que retrata, ao mesmo tempo, a estrutura regional e os diferentes subgrupos como variáveis externas.

\section{RESULTADOS ESPERADOS}

Pela via proposta, espera-se que os resultados produzidos possam aprimorar, no que for possível, os aportes teórico-conceituais relacionados com o objeto de estudo, a cor em ambientes de trabalho de escritório, ampliando sua abrangência analítica, bem como favorecendo o desenvolvimento de projetos balizados por evidências empíricas que priorizam a cognição das pessoas.

Espera-se, ainda, responder diretamente a todos os objetivos formulados que, em termos gerais, pretende apresentar uma proposta para avaliar a qualidade cromática de ambientes 


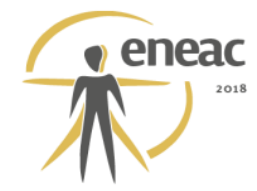

de trabalho de escritório a partir da percepção de especialistas e não especialistas no projeto desses espaços. Busca-se ainda, mais especificamente, testar a aderência de certas características visuais de ambientes de trabalho de escritório para a avaliação da sua qualidade cromática percebida; verificar a influência dessas características nos aspectos de agradabilidade, emoção e relaxamento nesses ambientes e, finalmente, analisar o consenso dos resultados entre os dois diferentes grupos abordados.

Os achados poderão confirmar as características dos ambientes de trabalho de escritório determinantes para a avaliação da sua qualidade cromática percebida, os efeitos da complexidade e da coerência dos ambientes de trabalho de escritório na percepção de sua qualidade cromática e, finalmente, demonstrar se trabalhadores, arquitetos e designers de interiores compartilham respostas avaliativas ou estéticas. Será, pois, possível compreender como diferentes configurações de ambientes de trabalho de escritório podem ser obtidas para diversos fins, ou seja, como criar um escritório agradável, emocionante, relaxante.

Em última análise, espera-se que os arquitetos e designers de interiores poderão fazer bom uso dos dados empíricos produzidos através dessa pesquisa, no sentido de obter bases científicas seguras para embasar suas decisões projetuais. Nessa linha, a abordagem proposta poderá, ainda, contribuir para a melhoria da qualidade cromática percebida de ambientes de trabalho de escritório.

\section{REFERÊNCIAS}

BINS Ely, V. Ergonomia + Arquitetura: buscando um melhor desempenho do ambiente físico. In: 3o ERGODESIGN - 3o CONGRESSO INTERNACIONAL DE ERGONOMIA E USABILIDADE DE INTERFACES HUMANO -TECNOLOGIA. 2003. Rio de Janeiro. Anais... Rio de Janeiro, LEUI/PUC Rio, 2003.

BERLYNE, D. E. Ends and meanings of experimental aesthetics. Canadian Journal of Psychology, 26, 1972. p 303-325.

BILKSY, W. (2003). A Teoria das Facetas: noções básicas. Estudos de Psicologia, 357-365.

CANTER, D.; BROWN, J.; GROAT, L. A multiple sorting procedure for studying conceptual systems, In: BRENNER, M; BROWN, J.; CANTER, D. (Eds). The research interview: uses and approaches. London: Academic Press, 1985. 79- 114.

COSTA FILHO, L. L. O enfoque da teoria das facetas na avaliação de lugares. In: V ENEAC ENCONTRO NACIONAL DE ERGONOMIA DO AMBIENTE CONSTRUÍDO E VI SEMINÁRIO NACIONAL DE ACESSIBILIDADE INTEGRAL, 2014. Rio de Janeiro. Anais... Rio de Janeiro, PUCRio, LEUI/PUC - Rio. 2014.

COSTA FILHO, L.; OLIVEIRA, I. F.; YOKOYAMA, S. A. A qualidade percebida em cenas do comércio varejista do centro de caruaru. In: VI ENEAC - ENCONTRO NACIONAL DE ERGONOMIA DO AMBIENTE CONSTRUÍDO E VII SEMINÁRIO NACIONAL DE ACESSIBILIDADE INTEGRAL, 2016. Recife. Anais... Recife, UFPE, 2016. p. 541-552

FIGUEIREDO, J.; MONT'ALVÃO, C. Cor nos locais de trabalho: como aplicá-la de forma adequada às necessidades dos usuários e às exigências da tarefa. In: XIV CONGRESSO BRASILEIRO DE ERGONOMIA, n. 14, 7f, out/nov. 2006. Curitiba. Anais... Curitiba, ABERGO, 2006.

A ergonomia ambiental no processo de composição cromática de locais de trabalho de escritório. In: MORAES, A. (Org.). Ergodesign do ambiente construído e habitado: ambiente urbano, ambiente público, ambiente laboral. Rio de Janeiro: iUsEr, 2004. p109-134. 
KAPLAN, Stephen. Perception and landscape: conceptions and misconceptions. In NASAR, J. Environmental aesthetics: Theory, research, and application. New York: Cambridge University Press, 1988. p.45-55.

MAHNKE, F. Color, environment \& human response: an interdisciplinary understanding of color and its use as a beneficial element in the design of the architectural environment. 1. Ed. New York: John Wiley \& Sons, 1996.

MONTEIRO, C. M. G.; ROAZZI, A. Polemic images: Dwellers' concepts of life in historic areas. In: COHEN, A. (Ed.). Facet Theory and scaling: in search of structure in behavioral and social sciences. Israel: Rubin R. I. D, 2009.

NASAR, J. L. The evaluative image of places. In: WALSH, W. B.; CRAIK, K. H.; PRINCE, R. H. 2nd. ed. (Eds.). Person-environment psychology: new directions and perspectives. New Jersey: Lawrence Erlbaum Associates, 2000. p. 117-168.

The effect of sign complexity and coherence on the perceived quality of retail scenes. In: NASAR, J. L. (Ed.). Environmental Aesthetics: theory, research, \& applications. New York: Cambridge University Press, 1988. p. 300-320.

REIS, T. C. dos; MORAES, A. Contribuição da ergonomia em projeto de espaço de trabalho. In: MORAES, A. (Org.). Ergodesign do ambiente construído e habitado: ambiente urbano, ambiente público, ambiente laboral. Rio de Janeiro: iUsEr, 2004. p.135-145.

RUSSEL, J. Affective appraisals of environments. In: NASAR, J. (Ed.). Environmental aesthetics: theory, research, and application. New York: Cambridge University Press, 1988. p. 120-129.

ROAZZI, A.; DIAS, M. G. B. B. Teoria das facetas e avaliação na pesquisa social transcultural: Explorações no estudo do juízo moral. In: Conselho Regional de Psicologia - 13a Região PB/RN (Ed.). A diversidade da avaliação psicológica: considerações teóricas e práticas. João Pessoa: Idéia, 2001. p.157-190

SHYE, S.; ELIZUR, D.; HOFFMAN, M. Introduction to Facet Theory: Content design and intrinsic data analysis in behavioral research. London: Sage, 1994.

SILVA JÚNIOR, J. A.; COSTA FILHO, L. L. Proposta para a avaliação da qualidade percebida de vitrinas. In: V ENEAC - ENCONTRO NACIONAL DE ERGONOMIA DO AMBIENTE CONSTRUÍDO E VI SEMINÁRIO NACIONAL DE ACESSIBILIDADE INTEGRAL, 2016. Recife. Anais... Recife, UFPE, 2016.

SILVA, V. L. A.; BORMIO, M. F. A importância do uso ergonômico da cor na interface ambiente $x$ usuário. In: I CONAERG - CONGRESSO INTERNACIONAL DE ERGONOMIA APLICADA, v. 3, n. 3, p., nov/dez. 2016, Recife. Anais... Recife, UFPE, 2016.

STAMPS, A. E. Perceptual and preferential effects of photomontage simulations of environments. In: Perceptual and Motor Skills, № 74, 1992.

WOHWILL, J. F. Environmental aesthetics: The environment as a source of affect. In: ALTMANN, I.; WOHWILL, J. F. (Eds.), Human Behaviour and Environment, V. 1, 1976. p 37-86. 